\title{
Health Status Monitoring of Cuplock Scaffold Joint Connection Based on Wavelet Packet Analysis
}

\author{
Liuyu Zhang, ${ }^{1,2}$ Chenyu Wang, ${ }^{2}$ and Gangbing Song ${ }^{2}$ \\ ${ }^{1}$ Engineering Research Center for Large Highway Structure Safety of the Ministry of Education, Changan University, \\ Xian, Shaanxi 710064, China \\ ${ }^{2}$ Smart Material and Structure Laboratory, Department of Mechanical Engineering, University of Houston, Houston, TX 77004, USA
}

Correspondence should be addressed to Gangbing Song; gsong@uh.edu

Received 8 August 2014; Accepted 13 October 2014

Academic Editor: Bo Chen

Copyright (C) 2015 Liuyu Zhang et al. This is an open access article distributed under the Creative Commons Attribution License, which permits unrestricted use, distribution, and reproduction in any medium, provided the original work is properly cited.

\begin{abstract}
The cuplock scaffolds are widely used as a temporary facility in the bridge and other constructions. The looseness of cuplock scaffold connection plays a significant role in the stability of the structure. Contemporary structural health monitoring method is reviewed in this paper at first, and then we proposed wavelet packet analysis based method. In order to detect the looseness of cuplock connection, three stress PZTs and three shear PZTs used as transducers are mounted onto vertical bar and cross bars of cuplok scaffold. Wavelet packet analysis is applied to analyze the transmitted signal energy between two PZTs through cuplok connection. Experimental results show that shear PZT has better performance than stress PZT to be used as actuator and sensor for the wavelet packet analysis based structural health monitoring. Besides, a sensor looseness index matrix (SLIM) is derived to indicate the looseness of the cuplock connection. The experimental results show that looseness index increases as the connection gets loose.
\end{abstract}

\section{Introduction}

Scaffolds are widely used in the construction of permanent works such as buildings and bridges. There are two major types of scaffold structures: one is made from prefabricated components and the other is made from steel tubes or bamboo. Cuplock scaffold is one of the scaffold structures which are made from prefabricated components. Cuplock scaffolds are often used in the US and Asia. In China, cuplock scaffolds are promoted promptly in recent years due to their low cost to manufacture, simplicity to assemble and disassemble, and high load capacity. Cuplock scaffold structure is only used as a temporary facility in construction, so the safety issues are overlooked sometimes. Some serious collapse accidents happened in China during the last several years [1-3]. It attracts more and more attention of researchers to the stability and safety issues of cuplock scaffolds.

The scaffold collapses are caused by overload, geometric imperfections, joint connections, and foundation failures [47]. Many researchers have carried out a lot of studies on these factors affecting the safety of scaffold structures. The causes of scaffold collapses were analyzed from eccentric compression of the vertical bar, materials, and management in [8]. The authors obtained the measurements of geometric imperfections and loading eccentricity of cuplock support scaffold systems from various construction sites in Sydney area and presented a study of cuplock scaffold systems joint stiffness [9]. The researchers investigated the effects of uncertainties on the ultimate strength of multistorey steel support scaffold frames through a rational statistical framework and a secondorder inelastic finite element analysis [10]. The mode of failure, effects of different random variables on the variability of structural strength, and the reliability of the scaffold structures were investigated in [11]. The authors developed accurate three-dimensional advanced analysis models to capture the behavior of support scaffold systems and proposed methods for modeling spigot joints, semirigid upright-to-beam connections, and base plate eccentricities [12]. The scholars presented an algorithm to model scaffold behavior and follow the full moment-rotation curve including nonlinear loading and unloading behavior and including looseness and obtained that for sway frames looseness makes the frame 
unstable but for the braced frame analyzed looseness had little effect on the result in [13]. Nevertheless, no work about the monitoring of the tightness of cuplock connection was done. In the field of construction, the joints will get loose if the workers forget to tighten the top cuplock when the joints are assembled. The joints can also be loose because of the vibration during the construction process. Then a relatively large gap will take place between the vertical bar and the cross bar. So the structural characteristics of scaffold will be essentially changed as well as the parameters of buckling. The author summarized the causes of 120 failures predominantly in the UK with approximately $30 \%$ of the failures that happened during construction [14]. Of the known causes of collapse, approximately $40 \%$ of the collapses occurred during pouring of concrete $[4,15]$. The majority of the scaffold accidents were caused by faulty platforms including platform supports, human error, unsafe working procedures, and faulty access arrangements. In [6], it was presented that the majority of the scaffold failures occurred due to poor site control. Since the instability and failure of structures are very likely to happen, the monitoring of the tightness for cuplock connection is very important.

Structural health monitoring (SHM) is widely used to monitor the real time status of the structure. It is a very effective way to detect the status of pipeline network. The reliability and safety of the pipeline network can be improved by using SHM applications [16]. Piezoelectric lead zirconate titanate (PZT) based approach is always used as transducer in active monitoring of the structure for its great features such as the availability of different shapes, broadband response frequency, low price, and the ability of being employed as actuator or sensor [17]. A new technology called smart aggregate that uses embedded piezoceramic based transducers has been applied to monitor cracks in concrete structures [1820]. However, SHM with PZT has not been used in detecting the looseness status of cuplock scaffolds by researchers. To monitor the status of a cuplock connection, we propose a SHM system in which PZTs are used as transducers. Two types of PZTs, stress PZT and shear PZT, are commonly used. In our experiment, we use both types of PZT and compare their performance for our scenario. Swept sine wave is used as an excitation, and the signal transmits through the cuplock connection between two PZTs. By using wavelet packet analysis, we can find the relation between the tightness degree and increase of transmitted signal.

Wavelet packet analysis is used as a signal-processing tool to analyze the sensor signal of the embedded PZT patches in the concrete structure and also pipe networks. We use wavelet packet analysis for the reason that it makes the analysis of signal which has narrow frequency bands and short time period available. Various kinds of damage indices have been developed for health monitoring of civil structures in recent years. Root-mean-square deviation (RMSD) is one of the commonly used damage indexes to compare the difference between the signatures of healthy and damaged states. This method can also be used in our analysis. RMSD is used to calculate the looseness index which is for comparing the difference between tightness status and looseness status.
The rest of the paper is organized as follows. In Section 2, the detection principle for cuplock connection looseness status is presented. The experimental setup is introduced in Section 3. In Section 4, the experiments are conducted to validate the relation between the looseness degree and energy of transmitted signal. Concluding remarks are drawn in Section 5.

\section{Detection Principle for Cuplock Connection Loosening Based on Wavelet Packet Analysis}

2.1. Cuplock Scaffold Structure. The cuplock scaffold is a versatile shoring system used to support formwork. The construct of cuplock scaffold members is shown in Figure 1 [21]. It consists of the vertical bar, cross bar, joints of cross bar, top cuplock, bottom cuplock, and locating dowel pin. The top cuplock can be moved along the vertical bar. By tightening the top cuplock, cross bars can be connected to the vertical bar firmly. The maximum number of cross bars that can be installed at one node is four.

2.2. Active Monitoring Principle Using Piezoelectric Transducers. The cross section of cuplock connection is shown in Figure 2. By tightening the top cuplock, a well-fitted cuplock connection with enough contact can be made. In order to maintain the carrying capacity and stability of cuplock scaffold, there must be enough pressure formed on the contact surface. Moreover, with the increase of cuplock connection tightness, the connection region could be larger and harder pressed.

Two pieces of PZTs are stuck on the cross bar and the vertical bar, respectively, as sensors and an actuator (as shown in Figure 3). PZT 3 (actuator) generates a wave that propagates across the cuplock connection, and then the signal is captured by PZT 1 and PZT 2 (sensor). Between the actuator and the sensor, energy is transmitted through pipes by the propagated waves. Input signal attenuate significantly at cuplock connection for the energy is lost there. There would be less energy of the input signal transmitted through cuplock connection when the connection gets looser and looser. The lost energy is correlated to the tightness of cuplock connection. The PZT can be not only stress PZTs but also shear PZTs.

\subsection{Detection Principle for Cuplock Connection Based on} Wavelet Packet Analysis. One of the useful methods to analyze the transmitted signal between two PZTs is wavelet packet analysis. A waveform that has an average value of zero in a limited duration can be called a wavelet. In wavelet analysis, a signal can be divided into two parts, low frequency and high frequency, which are approximation and detail, respectively. The approximation itself can be divided into a second level approximation and detail then. The detail can also be split the same way as approximation in wavelet packet analysis. The outstanding feature of wavelet packet analysis is that it makes inspection of relatively narrow frequency bands during a relatively short time window possible. 

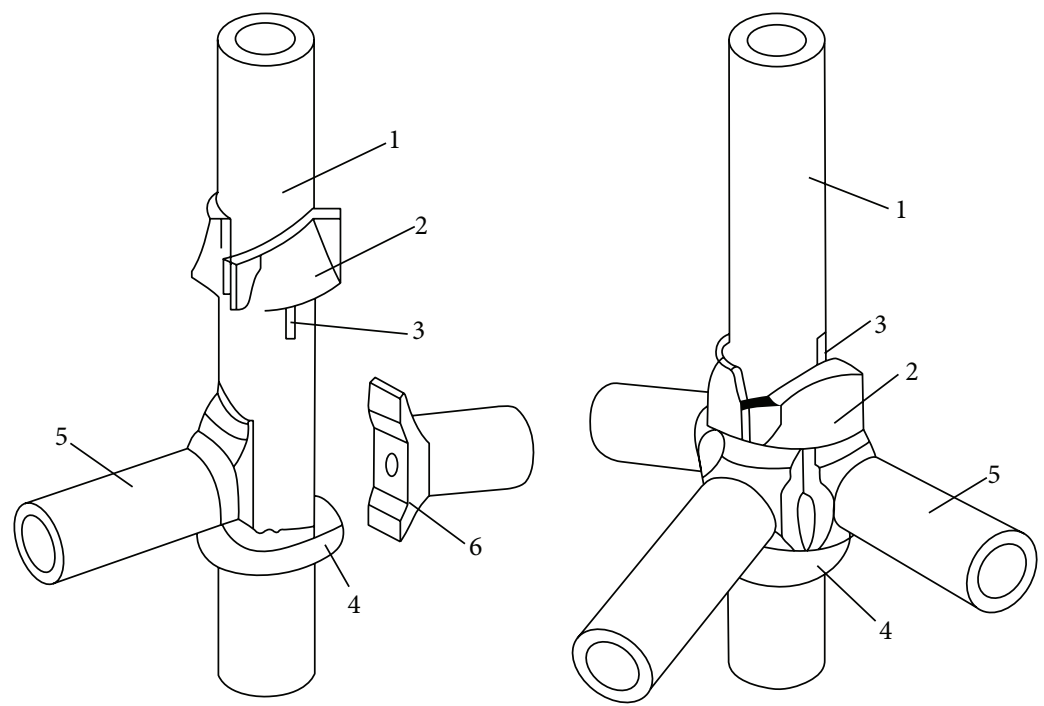
(1) Vertical bar
(2) Top cuplock
(4) Bottom cuplock
(3) Locating dowel pin
(5) Cross bar
(6) Cross bar joint

FIGURE 1: The construct of cuplock scaffold members.

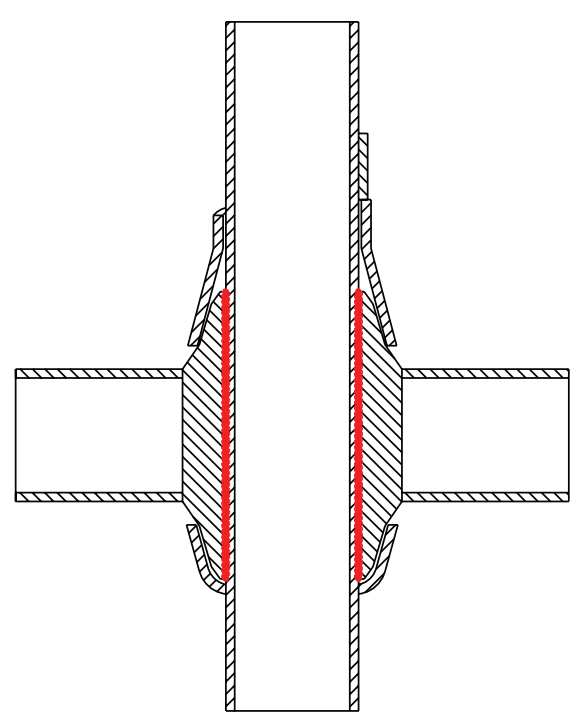

Figure 2: Cross section of cuplock connection.

The sensor signal $S$ is decomposed by an $n$-level wavelet packet decomposition into $2^{n}$ signal sets $\left\{X_{1}, X_{2}, \ldots, X_{2^{n}}\right\}$. $E_{i, j}$ is the energy of the decomposed signal, where $i$ is the tightness index and $j$ is the frequency band $\left(j=1, \ldots, 2^{n}\right)$. $X_{j}$ can be expressed as

$$
X_{j}=\left[x_{j, 1}, x_{j, 2}, \ldots, x_{j, m}\right],
$$

where $m$ is the amount of sampling data. Energy of the decomposed signal is defined as

$$
E_{i, j}=\left\|X_{j}\right\|_{2}^{2}=x_{j, 1}^{2}+x_{j, 2}^{2}+\cdots+x_{j, m}^{2} .
$$

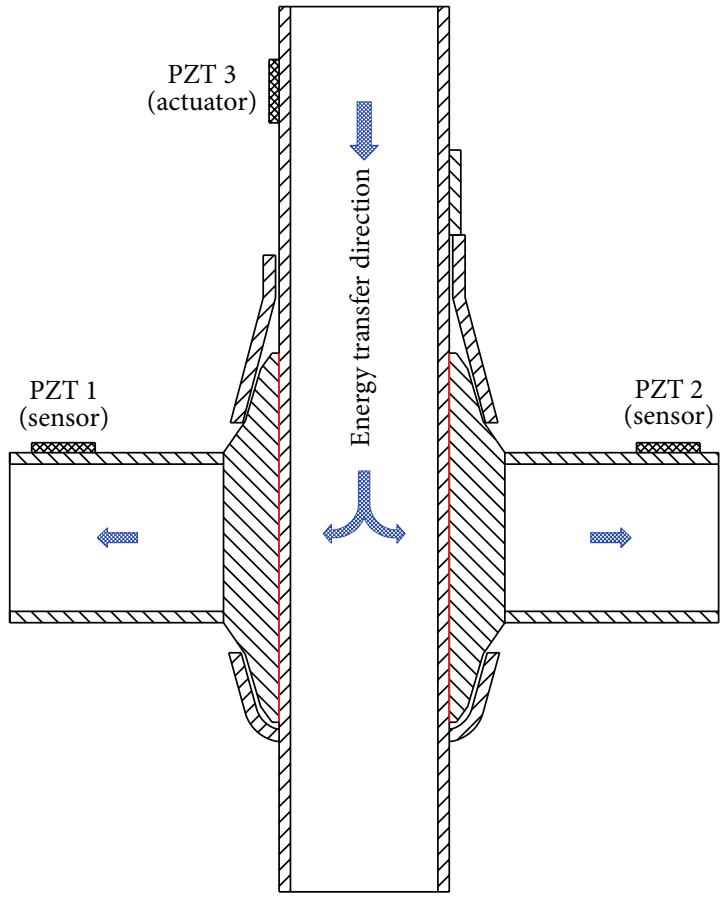

Figure 3: Energy transmission at the cuplock scaffold model.

Energy vector at tightness index $i$ is defined as

$$
E_{i}=\left[E_{i, 1}, E_{i, 2}, \ldots, E_{i, 2^{n}}\right] .
$$

We use root-mean-square deviation (RMSD) as the tightness index to compare the difference between the features of tight and loose states. The tightness index is formed by calculating the RMSD between the energy vectors of the tight 


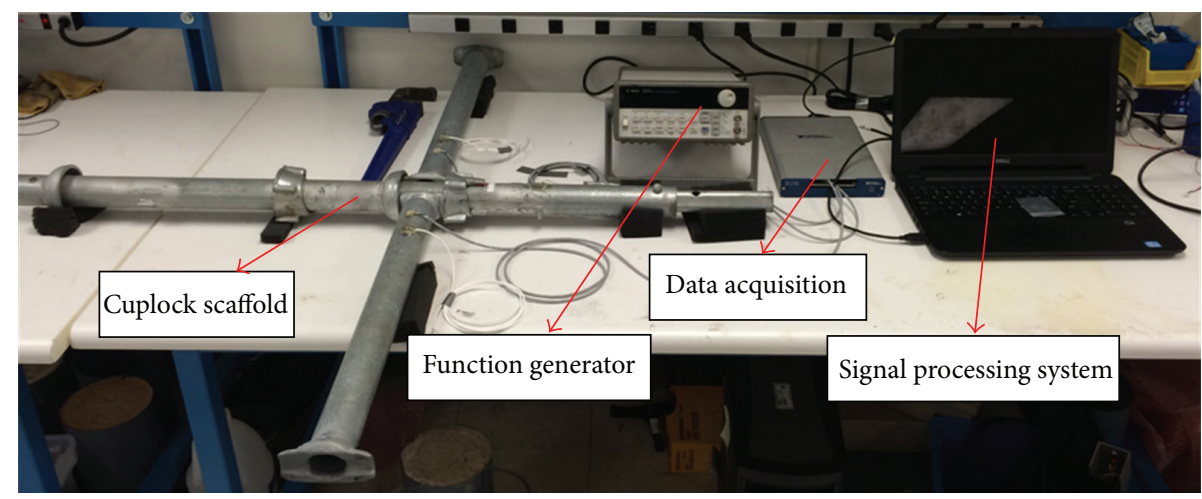

Figure 4: Experimental setup.

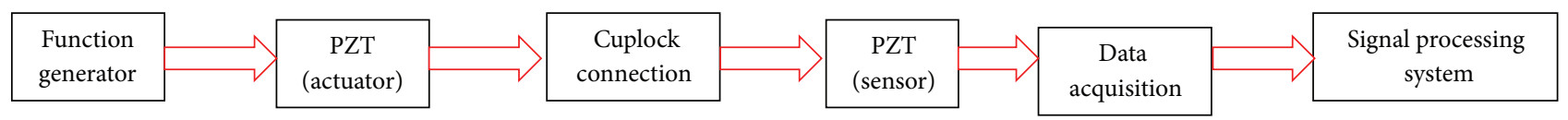

Figure 5: Experimental flow.

state and the loose state. The energy vector for tight data is $E_{t}=\left[E_{t, 1}, E_{t, 2}, \ldots, E_{t, 2^{n}}\right]$. The energy vector $E_{i}$ for the tight state at tightness index $i$ is defined as $E_{i}=\left[E_{i, 1}, E_{i, 2}, \ldots, E_{i, 2^{n}}\right]$. The looseness index at tightness index $i$ is defined as

$$
I=\sqrt{\frac{\sum_{j=1}^{2^{n}}\left(E_{i, j}-E_{t, j}\right)^{2}}{\sum_{j=1}^{2^{n}} E_{t, j}^{2}}}
$$

The proposed looseness index represents the transmission energy loss portion caused by looseness of cuplock connection. The connection is tight when the looseness index is close to 0 . When the looseness index is above a certain threshold, the cuplock connection is not tight anymore. In this case, the instability of the structure increases as the looseness index increases.

To demonstrate the tight status at different tightness levels, one type of looseness index matrix is defined, a tightness-level looseness index matrix.

A sensor looseness index matrix (SLIM) is as follows.

$M_{m \times n}$ is defined as

$$
M_{m \times n}=\left[I_{i, j}\right] \quad(i=1, \ldots, m, j=1, \ldots, n),
$$

where the matrix element at the $i$ th row and $j$ th column, $I_{i, j}$, is the looseness index of the $i$ th PZT at time of $j$ th test, $m$ is the total number of tightness degrees, and $n$ is the total number of PZT sensors. The looseness status at different locations of the scaffold specimen at different tightness degrees can be described by a two-dimensional looseness index matrix. The SLIM is useful in monitoring the looseness evolution process to predict the failure of a structure. For a specific location, you can compare the looseness index with the tightest one to get the looseness of the connection from SLIM.
TABle 1: Parameters for swept sine wave.

\begin{tabular}{lc}
\hline Parameters & Value \\
\hline Frequency & $100 \mathrm{~Hz} \sim 150 \mathrm{KHz}$ \\
Amplitude & $10 \mathrm{~V}$ \\
\hline
\end{tabular}

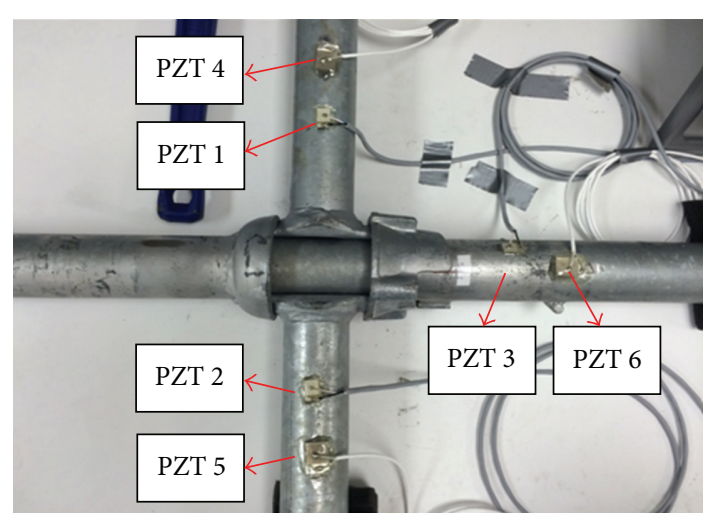

FiguRE 6: Cuplock scaffold structure specimen.

\section{Experimental Setup}

In Figure 4, it is the experimental setup for active monitoring of a cuplock connection. The experiment flow is shown in Figure 5. The PZT actuator is excited by the swept sine wave (Table 1) which is generated by function generator. Upon excitation, the actuator generates waves that propagate through the cuplock connection. Then the wave will be received by the PZT sensor. We use the data acquisition system to sample the signal sensed by PZT sensor at the rate of $1 \mathrm{MHz}$ and finally use the signal-processing system in the computer to analyze the signal data. The signal received by the sensors can indicate the looseness of the connection. The cuplock scaffold model and six PZTs are shown in Figure 6. 
TABLE 2: Cuplock Scaffold model features.

\begin{tabular}{lcccc}
\hline & $\begin{array}{c}\text { Diameter } \\
(\mathrm{mm})\end{array}$ & $\begin{array}{c}\text { Wall thickness } \\
(\mathrm{mm})\end{array}$ & $\begin{array}{c}\text { Yield strength } \\
(\mathrm{MPa})\end{array}$ & Elongation \\
\hline Vertical bar & 48.26 & 3.175 & 345 & $30 \%$ \\
Cross bar & 48.26 & 3.175 & 345 & $30 \%$ \\
\hline
\end{tabular}

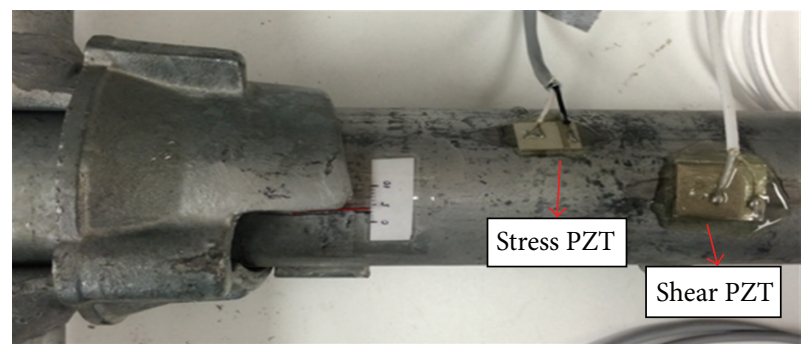

Figure 7: Picture of tightness scale and mounted stress PZT and shear PZT.

This cuplock scaffold model is composed of a vertical bar and two cross bars. The features of the cuplock specimen are listed in Table 2. Two cross bars are connected with the vertical bar through the cuplock. The PZTs are mounted on the outside surface of two cross bars and the vertical bar. Figure 7 is the photo of PZT patch transducers on the vertical bar. We use nonconductive epoxy to stick each PZT patch onto the pipe surface. Nonconductive epoxy is also used to protect PZTs from damage by covering their top. We mount a plastic point to the top cuplock and $1 \mathrm{~cm}$ ruler on the vertical bar as a scale which shows tightness index $i$ (Figure 7).

There are two types of PZTs used in our experiment, three stress PZTs (PZT 1, 2, and 3) and three shear PZTs (PZT 4, 5, and 6). We have experiment sets 1 and 2 with stress PZTs and shear PZTs, respectively. In experiment 1 , the actuator is PZT 3 and the sensors are PZTs 1 and 2. PZT 6 is the actuator for experiment 2 , and PZTs 4 and 5 are sensors. The performance of two sets of experiments is compared.

\section{Experimental Results and Analysis}

Two sets of experiments were done during the cuplock connection monitoring test. In these experiments, we used pipe wrench to screw the connection by $1 \mathrm{~mm}$ each time from tightness degree 3 to 6 . The tightness degree 3 to 6 are denoted by tightness index 1 to 4 . The lowest tightness degree 3 is chosen for the reason that it is the degree that can be tightened by hand. The highest tightness degree 6 is the degree that can be tightened by the pipe wrench. These two degrees are chosen based on the practical field operation conventions. When the workers build the cuplock structure in field, the first step for them is to tighten the cuplock connection by hand and then use hammer to tighten it.

The PZTs are utilized for the structure monitoring of the cuplock scaffold specimen. In the structural health monitoring algorithm, wavelet packet analysis is used as a signalprocessing tool to analyze the sensor signal. The sensor signal is decomposed by a 5-level wavelet packet decomposition

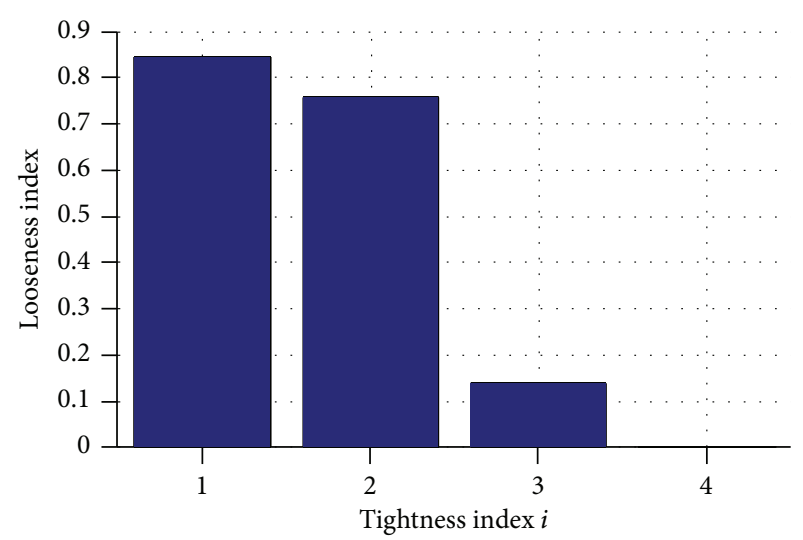

Figure 8: Looseness index (PZT 3 as actuator and PZT 1 as sensor).

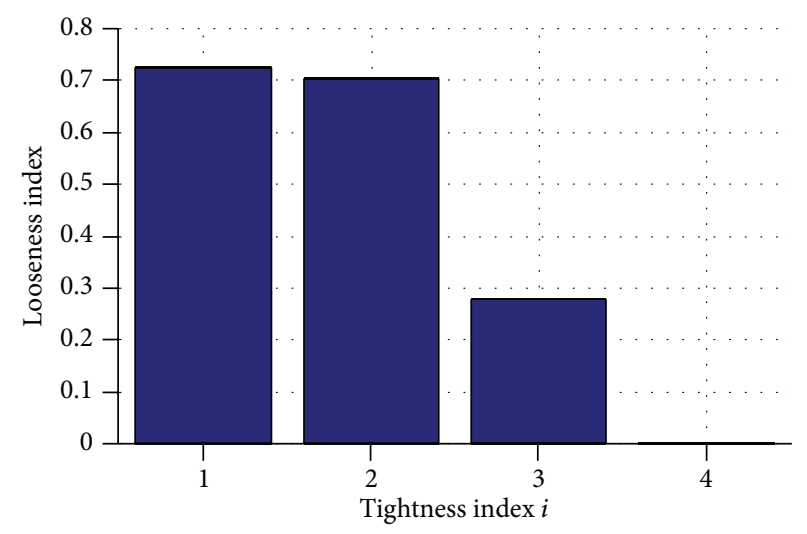

FIgURE 9: Looseness index (PZT 3 as actuator and PZT 2 as sensor).

into 32 signal sets. 32 signal sets are enough for the analysis. The wavelet packet based looseness index matrix proposed previously has been utilized for the cuplock scaffold structure looseness monitoring.

4.1. Experiment Set 1 with Stress Wave PZTs. Figures 8 and 9 are the looseness index graphs in which the data is sensed by PZTs 1 and 2, respectively, for stress PZTs. In the experiment, tests were done in the same condition. So we compare the results of Figures 8 and 9 at first. The difference of the looseness for tightness indexes 1 and 2 is little. It is because the contact surface of the cuplock scaffold specimen is not smooth, so when the cuplock was tightened the transmitted signal energy did not change much. It also may be caused by the characteristic of stress wave; when the contact area is not enough, the transmitted signal energy does not change much. When the cuplock got tightened from tightness 2 to tightness 3 , the difference is very significant.

4.2. Experiment Set 2 with Shear Wave PZTs. Figures 10 and 11 are the looseness index graphs in which the data is sensed by PZTs 4 and 5, respectively, for shear PZTs. In the experiment, tests were done in the same condition. Comparing the results of Figures 10 and 11, we found the loose index changes gradually from tightness degree 1 to 4 . The possible reason is 


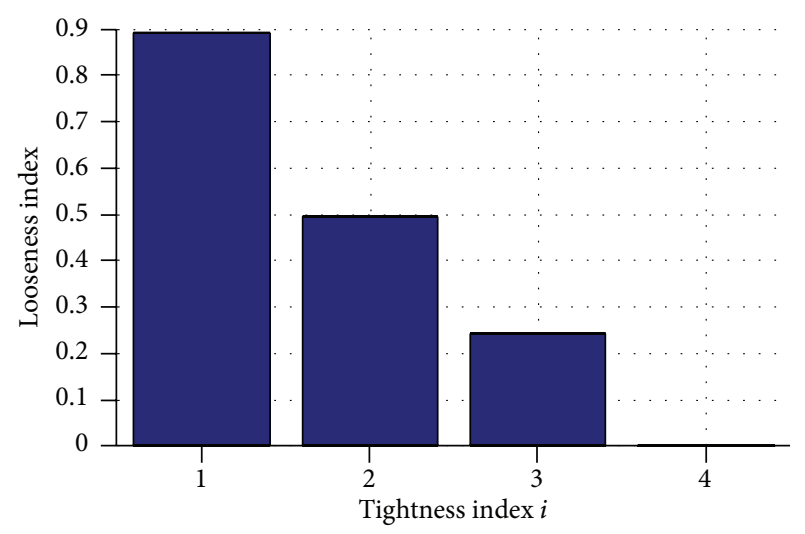

Figure 10: Looseness index (PZT 6 as actuator and PZT 4 as sensor).

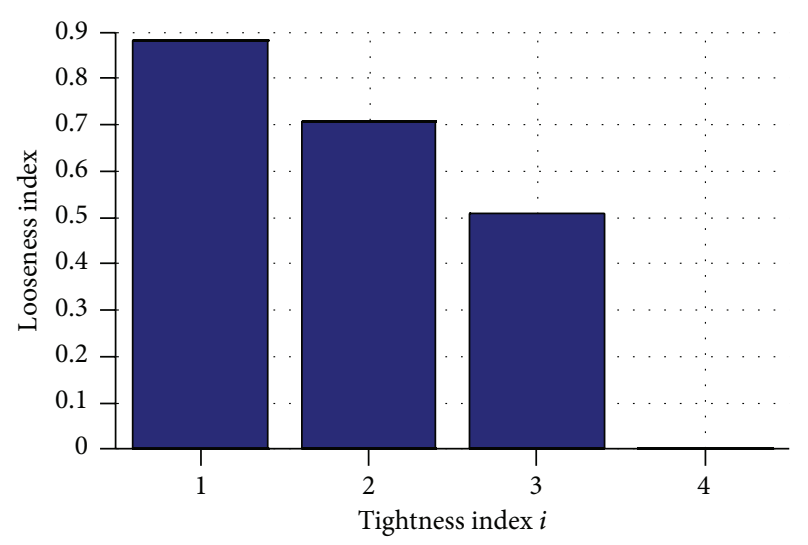

FIGURE 11: Looseness index (PZT 6 as actuator and PZT 5 as sensor).

that those shear waves transmit through the specimen well. The transmitted signal energy increased continuously with the incensement of the tightness index.

For both experiments, we can find that both stress and shear PZTs can be used to monitor the looseness of the cuplock connection. Comparing the results of experiments 1 and 2, we find that the shear PZTs work better than stress PZTs in our experiments. It is better to use shear PZTs to monitor the health status of cuplock connections in the construction field.

\section{Conclusions}

In this paper, we used piezoelectric transducers to monitor cuplock connection for cuplock scaffold based on the wavelet packet analysis. According to the experimental results, the looseness index decreased with the cuplock connection tightened. In summary, the looseness of cuplock connection can be monitored by the proposed method. The proposed active sensing method with piezoelectric transducers has the potential to be implemented in the health monitoring of structures with similar connections in construction.

\section{Conflict of Interests}

The authors declare that there is no conflict of interests regarding the publication of this paper.

\section{References}

[1] Y. Zhao and K. Liu, "Safety accident of formwork engineering and its prevention in china," Architecture Technology, vol. 39, no. 7, pp. 492-496, 2008 (Chinese).

[2] L. Ming and L. Kaiming, "Statistics and analysis of collapse accident of high scaffolding in china," Annual meeting of National Building formwork And Scaffolding Committee, vol. 36, pp. 80-84, 2009 (Chinese).

[3] J. Mi, "Cause and solution of scaffold collapse accident," Architecture Technology, vol. 32, no. 4, pp. 260-264, 2001 (Chinese).

[4] F. Hadipriono and H. K. Wang, "Causes of falsework collapses during construction," Structural Safety, vol. 4, no. 3, pp. 179-195, 1987.

[5] J. Peng, A. Pan, D. Rosowsky, W. Chen, T. Yen, and S. Chan, "High clearance scaffold systems during construction-I. Structural modelling and modes of failure," Engineering Structures, vol. 18, no. 3, pp. 247-257, 1996.

[6] R. G. Beale, "Scaffold research-a review," Journal of Constructional Steel Research, vol. 98, pp. 188-200, 2014.

[7] J. Xie and J. Wang, "Analysis and prevention technology measure of the collapse accidents of falsework system," Construction Technology, vol. 33, no. 2, pp. 35-37, 2004 (Chinese).

[8] W. K. Yu, K. F. Chung, and S. L. Chan, "Structural instability of multi-storey door-type modular steel scaffolds," Engineering Structures, vol. 26, no. 7, pp. 867-881, 2004 (Chinese).

[9] T. Chandrangsu and K. J. Rasmussen, "Investigation of geometric imperfections and joint stiffness of support scaffold systems," Journal of Constructional Steel Research, vol. 67, no. 4, pp. 576$584,2011$.

[10] H. Zhang, T. Chandrangsu, and K. J. R. Rasmussen, "Probabilistic study of the strength of steel scaffold systems," Structural Safety, vol. 32, no. 6, pp. 393-401, 2010.

[11] H. Zhang, K. J. R. Rasmussen, and B. R. Ellingwood, "Reliability assessment of steel scaffold shoring structures for concrete formwork," Engineering Structures, vol. 36, pp. 81-89, 2012.

[12] T. Chandrangsu, K. Rasmussen, and K. J. Rasmussen, "Structural modelling of support scaffold systems," Journal of Constructional Steel Research, vol. 67, no. 5, pp. 866-875, 2011.

[13] U. Prabhakaran, R. Beale, and M. Godley, "Analysis of scaffolds with connections containing looseness," Computers \& Structures, vol. 89, no. 212, pp. 1944-1955, 2011.

[14] A. Walker, Study and Analysis of the First 120 Failure Cases, Structural Failures in Buildings, The Institution of Structural Engineers, 1981.

[15] F. C. Hadipriono and H.-K. Wang, "Analysis of causes of falsework failures in concrete structures," Journal of Construction Engineering and Management, vol. 112, no. 1, pp. 112-121, 1986.

[16] F. Bouchoucha, M. Akrout, T. Fakhfakh, M. Ichchou, and M. Haddar, "Damage detection in cylindrical pipe through diffusion matrix in wave finite element method," Advances in Structural Engineering, vol. 15, no. 3, pp. 435-446, 2012.

[17] Z. Shi and T. Zhang, "Bending analysis of a piezoelectric curved actuator with a generally graded property for the piezoelectric parameter," Smart Materials and Structures, vol. 17, no. 4, Article ID 045018, 2008. 
[18] G. Song, Y. L. Mo, K. Otero, and H. Gu, "Health monitoring and rehabilitation of a concrete structure using intelligent materials," Smart Materials and Structures, vol. 15, no. 2, p. 309, 2006.

[19] G. Song, H. Gu, Y. L. Mo, T. T. C. Hsu, and H. Dhonde, "Concrete structural health monitoring using embedded piezoceramic transducers," Smart Materials and Structures, vol. 16, no. 4, pp. 959-968, 2007.

[20] G. Song, H. Gu, and Y.-L. Mo, "Smart aggregates: multifunctional sensors for concrete structures-a tutorial and a review," Smart Materials and Structures, vol. 17, no. 3, Article ID 033001, 2008.

[21] Y. Chen, L. Liu, and Y. Duan, "Study on the mechanical characteristic and safety measures of bowl-scaffold," ProcediaSocial and Behavioral Sciences, vol. 96, pp. 304-309, 2013. 

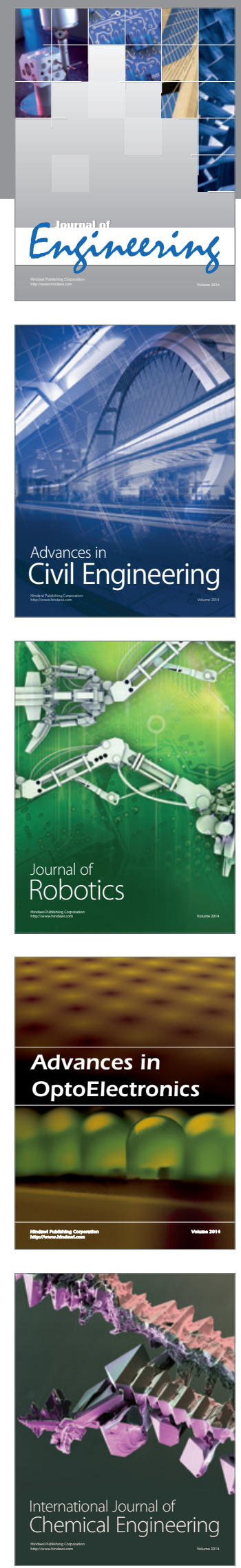

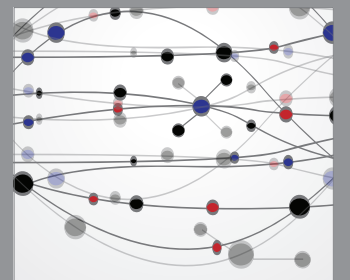

The Scientific World Journal
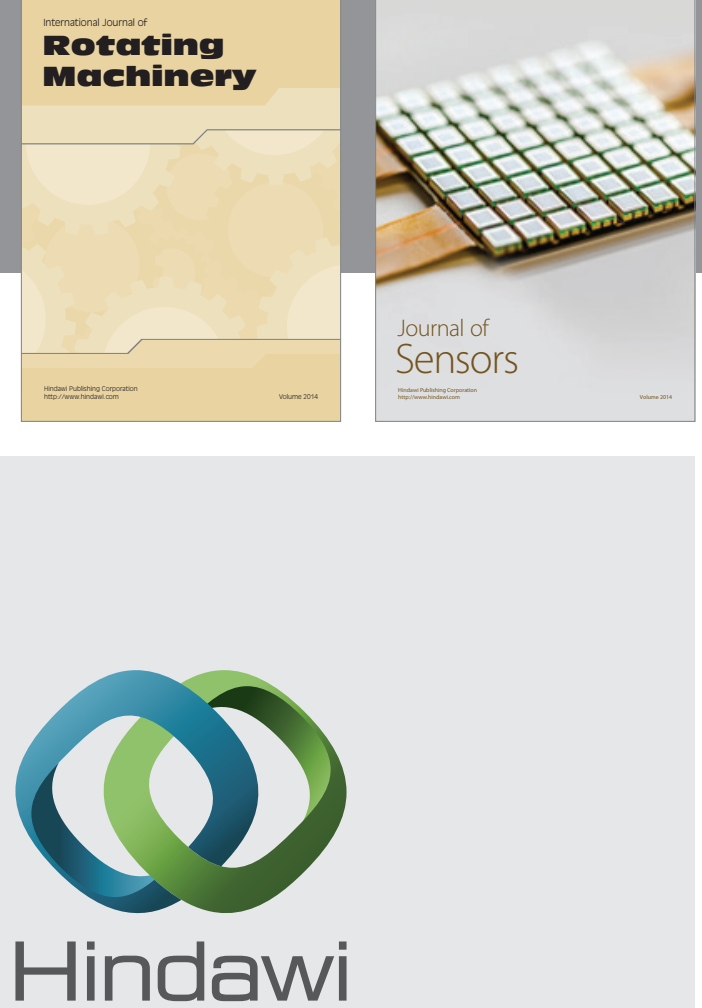

Submit your manuscripts at http://www.hindawi.com
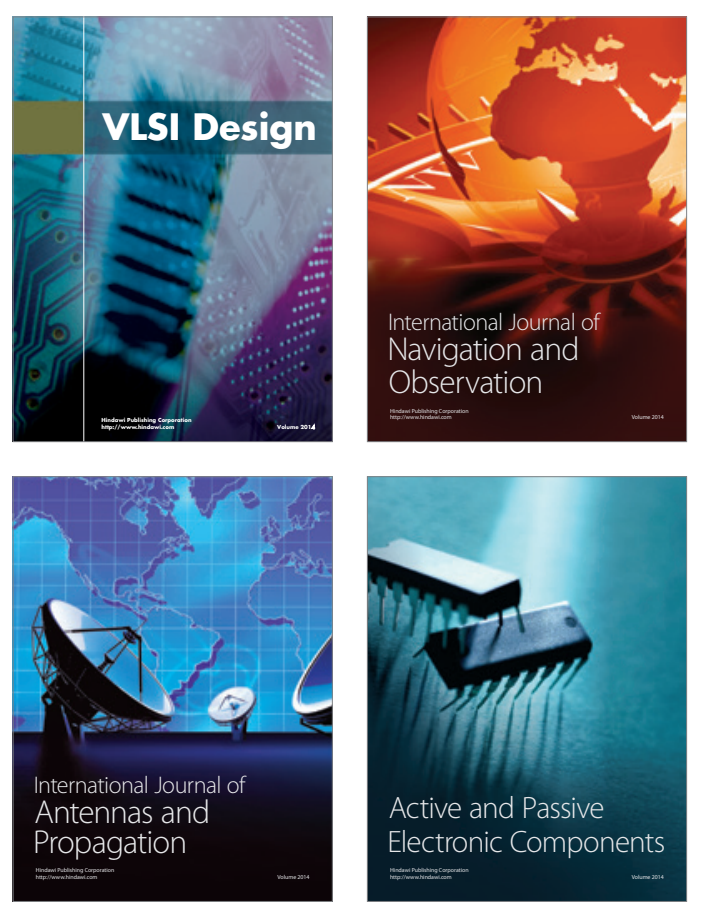
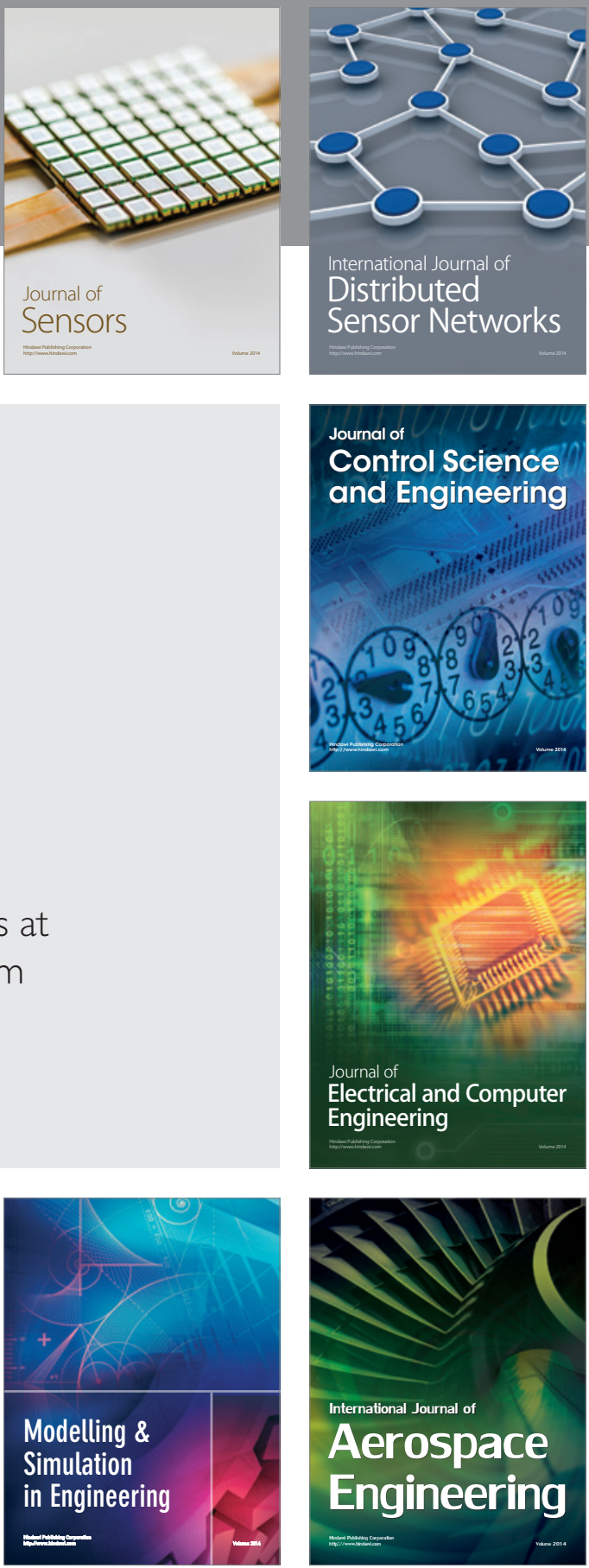

Journal of

Control Science

and Engineering
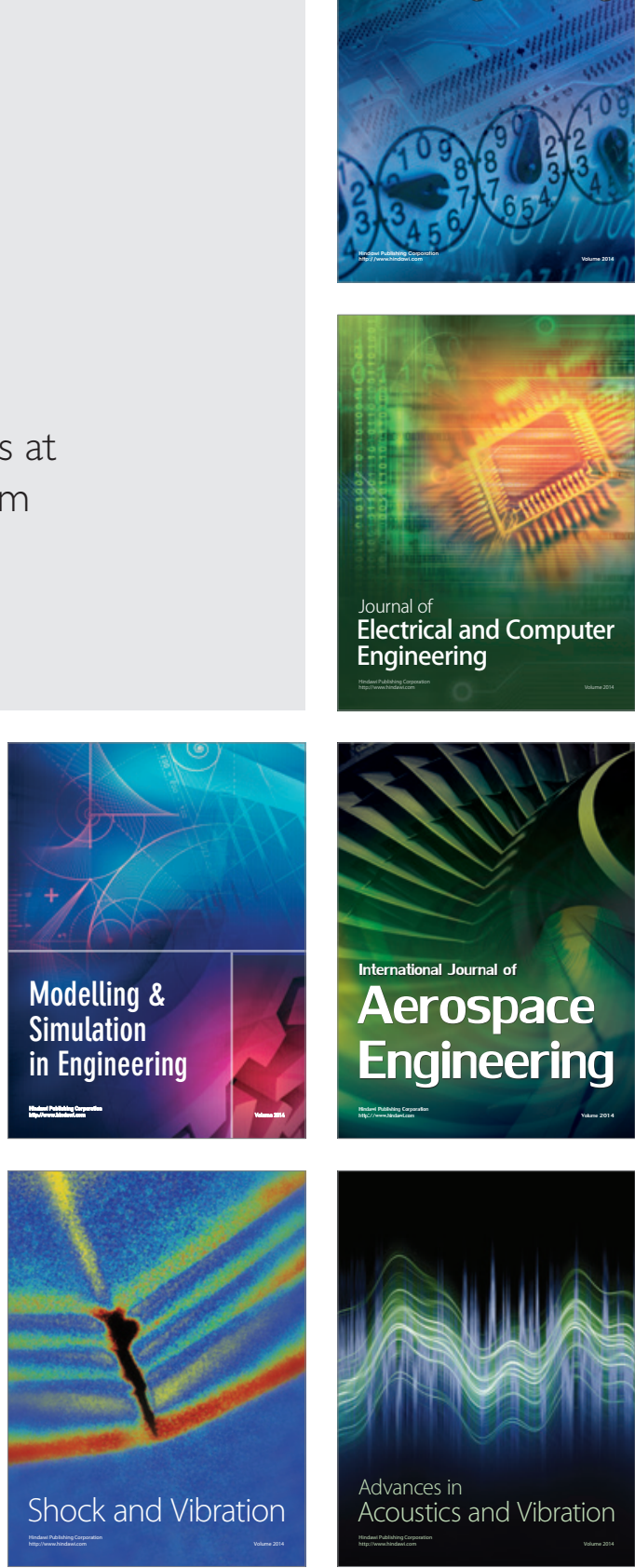